Published in final edited form as:

J Aging Health. 2018 March ; 30(3): 475-498. doi:10.1177/0898264316682916.

\title{
The Impact of Three Cognitive Training Interventions on Older Adults' Physical Functioning across Five Years
}

\author{
Lesley A. Ross, Ph.D. ${ }^{1}$, Briana N. Sprague, MS ${ }^{1}$, Christine B. Phillips, Ph.D ${ }^{1}$, Melissa L. \\ O'Connor, Ph.D. ${ }^{2}$, and Joan E. Dodson, Ph.D. ${ }^{3}$ \\ ${ }^{1}$ The Department of Human Development and Family Studies, The Pennsylvania State University, \\ University Park, PA 16802, USA \\ ${ }^{2}$ Department of Human Development and Family Science, North Dakota State University, Fargo, \\ ND 58108, USA \\ ${ }^{3}$ Psychology Department, University of Alabama at Huntsville, Huntsville, AL, 35816, USA
}

\begin{abstract}
OBJECTIVE-Physical functioning is closely associated with cognition. The current study assessed the impact of three cognitive training programs on objective measures of physical functioning across 5 years.
\end{abstract}

METHOD—Older adults randomized to a processing speed $(n=702)$, reasoning $(n=694)$, or memory $(n=703)$ training intervention were compared to those randomized to a no-contact control condition ( $n=698)$. Intention-to-treat (ITT) and treatment-received (time-varying number of training sessions) analyses were conducted.

RESULTS-There were no transfer effects in the ITT analyses. Treatment-received models demonstrated that training sessions (i.e., higher dosage) across all intervention arms transferred to better maintained Digit Symbol Copy and Turn 360 performance relative to the control group. More reasoning training transferred to better grip strength.

DISCUSSION-This is the first study to demonstrate differential longitudinal cognitive training transfer effects to three performance-based physical functioning measures. Future research should investigate mechanisms of far-transfer effects.

\section{Keywords}

Speed of processing training; Reasoning training; Memory training; Motor Function; Randomized controlled trial

Corresponding Author: Lesley A. Ross, The Pennsylvania State University, 115 Health and Human Development Building; University Park, PA 16802, Phone: +1 814-867-3189, Fax: +1 814-863-9423, 1ross@ psu.edu; lesleyaross@ gmail.com.

CONFLICTS OF INTEREST

The Authors declare that there is not conflict of interest. 


\section{Introduction}

Physical functioning, commonly recognized as the ability to carry out physical activities important for daily living, is a principal facet of health, well-being, and everyday performance (Painter, Stewart, \& Carey, 1999). Individuals with poor physical functioning are at greater risk for a variety of adverse outcomes including incident disability (Bohannon, 2008), functional decline (García-Peña et al., 2013), accelerated cognitive decline (AlfaroAcha, Snih, Kuo, Markides, \& Ottenbacher, 2006) and mortality (Al Snih, Markides, Ray, Ostir, \& Goodwin, 2002). Physical capacity is necessary to successfully navigate the environment and encompasses a number of components and motor abilities with differing degrees of complexity. Similarly, measures of physical functioning vary greatly and range from self-report to objective measures of motor movement abilities, such as muscular strength, locomotion, and coordination. For the purposes of this study, the broad term physical functioning is used to describe objectively measured motor movement abilities.

Increasing cross-sectional (Blankevoort et al., 2013) and longitudinal evidence (Clouston et al., 2013) has demonstrated that physical and cognitive functioning are strongly interrelated in advanced age. For example, better performance on lower limb function and balance, motor speed, grip strength, and composite physical functioning measures have been associated with better cognitive performance (Alfaro-Acha et al., 2006; Blankevoort et al., 2013; Bridenbaugh \& Kressig, 2015; Desjardins-Crépeau et al., 2014; Nieto, Albert, Morrow, \& Saxton, 2008; Rosano et al., 2005; van Iersel, Kessels, Bloem, Verbeek, \& Olde Rikkert, 2008). Motor functions have also distinguished older adults having no cognitive impairments from those with pre-clinical and clinical dementia (Kluger et al., 1997). Overall, consistent positive associations are found between physical functioning and cognition, particularly for fluid abilities such as executive function and processing speed (Anstey, Lord, \& Williams, 1997; Atkinson et al., 2010; Buchman, Boyle, Leurgans, Barnes, \& Bennett, 2011; Desjardins-Crépeau et al., 2014; van Iersel et al., 2008); see Clouston and colleagues (2013) for a review.

Pathways to age-related functional deficits are complex and not fully elucidated (Buchman et al., 2011). Proposed mechanisms range from specific age-related brain changes to a more global deterioration of brain integrity (Clouston et al., 2013). Observational studies using performance-based and self-report measures have provided mixed evidence. While some have suggested that cognitive functioning decrements may precede (Buchman et al., 2011) or cooccur with (Atkinson et al., 2010) physical functioning degradation, others indicate bidirectional associations (Gale, Allerhand, Aihie Sayer, Cooper, \& Deary, 2014). Experimental work examining practice on a dual task (postural control and memory retest) found that improvement on the memory task cooccurred with postural control improvement (Doumas, Rapp, \& Krampe, 2009). Taken together, these results suggest not only that cognitive and physical functioning are interrelated but also that cognitive processes may be part of a causal pathway to, or share a common mechanism with, physical functioning outcomes. Understanding not only the directionality of declines but also whether improvements in one functional domain will transfer to the untrained functional domain will help develop a better understanding potential mechanisms. 


\section{Cognitive Interventions and Physical Functioning}

Methods to enhance or maintain physical functioning often focus on rehabilitation or exercise programs (Gleeson, Sherrington, \& Keay, 2014; Lord et al., 2003), particularly those aimed at improving lower limb extremity strength and balance in at-risk populations. To our knowledge, only four studies have examined whether adaptive interventions that target cognitive abilities, which are closely connected to physical functioning in older adulthood, transfer to physical functioning. The first study (Li et al., 2010) examined the impact of 5 hours of computerized color decision and letter-identity visual discrimination task training on balance, mobility, and leg strength in a sample of 20 community-dwelling adults aged 70 and older. Results revealed immediate posttest improvements in standing balance but not gait speed or chair sit-to-stands. Verghese, Mahoney, Ambrose, Wang, and Holtzer (2010) found that 72 hours of Mindfit, a multi-domain cognitive training program, transferred to improved gait velocity (both normal walking and walking while talking), but not to normal pace walking, in a sample of 24 healthy sedentary older adults. Finally, SmithRay and colleagues $(2013 ; 2014)$ conducted two studies examining the transfer of Posit Science's Insight, a multi-domain cognitive training program, to physical functioning. The first study $(N=51)$ involved 30 hours of training across a 10 -week period and found transfer to better Timed Get Up and Go performance, but not gait speed, at posttest using intentionto-treat (ITT) analyses (2013). Further subsample analyses of participants with poor walking speed at baseline found both transfer effects and increased effect sizes for Timed Get Up and Go and distracted gait speed. The second Smith-Ray study examined the impact of 20 hours of the same multi-domain cognitive intervention across 10 weeks in Black older adults who had a balance impairment $(N=45)$. Results revealed significant transfer of the intervention to improved Berg Balance Scale and gait speed, but not distracted gait speed, at posttest (2014). Together, these four studies provide evidence in small samples that some adaptive cognitive interventions show potential in preventing gait- and balance-related mobility problems immediately after training.

Although these preliminary studies are promising, the effects of cognitive training on physical function remain unclear due to several important limitations (Albert, Bear-Lehman, $\&$ Anderson, 2015). First, the prior studies included small samples, which may have limited statistical power and generalizability (Smith-Ray et al., 2014). Second, as Smith-Ray and colleagues (2014) and others ( $\mathrm{Li}$ et al., 2010) noted, the studies only included immediate posttest, thus limiting the ability to examine maintenance effects, which are important from a public health perspective. Third, three of these studies (Smith-Ray et al., 2013; Smith-Ray et al., 2014; Verghese et al., 2010) used multi-domain interventions. While such interventions may increase the intrinsic motivation to train by preventing boredom and tap multiple important cognitive domains, use of such interventions limits the ability to examine selective effects of individual program components on specific cognitive domains or abilities. Investigation of the essential or most effective intervention components is an important part of current work examining mechanisms of training (Collins et al., 2014; Smith-Ray et al., 2014). That is, in a multi-domain intervention, one training component may be the primary or sole contributor to transfer effects (i.e., the "active ingredient"), but the omnibus effects may be diluted by less-effective or ineffective components (i.e., "inactive ingredients"). Finally, most of the work to date has focused on ITT or subsample 
analyses and has not included investigations regarding the dosage, or treatment-received, aspect of cognitive interventions (Ross et al., 2016; Smith-Ray et al., 2014). It is not only important to know how effective a training protocol is under real-world conditions, but it is also necessary to learn whether training effects increase with number of sessions. This would enable more efficiently designed protocols that could be tailored for specific outcomes. The present article addresses these limitations, and examines the impact of three separate cognitive interventions on objective measures of physical functioning across a 5year period in a multi-site sample of community-dwelling adults. Based on previous evidence, we hypothesized that speed of processing, reasoning, and memory training would transfer to performance on physical functioning tasks (Digit Symbol Copy, Turn 360, and grip strength), and that more pronounced effects would result from higher doses (i.e., more sessions) of training.

\section{Methods}

\section{Participants}

This study utilized secondary data from the Advanced Cognitive Training for Independent and Vital Elderly (ACTIVE) study, a multi-site, randomized clinical trial that investigated the effects of cognitive training on daily health and functional outcomes of 2,802 older adults over a five-year period. Inclusion criteria for the study were as follows: age 65 or older, visual acuity $\geq 20 / 50$, intact mental status (i.e., Mini-Mental State Examination score 223), no health conditions associated with cognitive impairment, sound verbal communication skills, no difficulties performing activities of daily living, and no recent participation in cognitive training. Eligible participants completed a baseline assessment and were then randomly assigned to a cognitive training or control condition. Five participants were removed from the sample due to incorrect randomization to booster training resulting in 2,797 participants randomized to the speed of processing training arm $(n=702)$, reasoning training arm $(n=694)$, memory training arm $(n=703)$, or a no-contact control arm $(n=698)$. At baseline, participants were an average of 73.6 years old $(S D=5.9)$ and reported 13.5 years of education $(S D=2.7)$. The sample was $75.8 \%$ female and $26.7 \%$ nonWhite. Descriptive statistics for each arm are displayed in Table 1. Extensive detail about ACTIVE can be found elsewhere (Jobe et al., 2001; Willis et al., 2006), ClinicalTrials.gov (Identifier NCT00298558).

\section{Study Design and Procedures}

ACTIVE includes an extensive number of primary and secondary outcomes, along with covariate measures, as detailed in Jobe and colleagues (2001). Baseline cognitive, physical functioning, lifestyle factors, and health assessments were conducted between 1998 and 1999. Participants were then randomized to one of three cognitive training arms or the nocontact control arm. The initial cognitive training interventions consisted of 10 sessions, 60-75 minutes each, administered over a 6-week period. Certified trainers conducted the training using standardized procedures with groups of three to four participants. Participants in all training conditions received feedback on their performance after each block of trials and experienced the same amount of social contact with the trainers. Follow-up assessments were conducted approximately 2 months (posttest), 1 year, 2 years, 3 years, and 5 years after 
baseline testing. Turn 360 and grip strength were not assessed at either the immediate posttest or the 1 year postintervention. Compliant trained participants (i.e., completed at least eight training sessions) were randomized to either receive an additional four booster sessions prior to assessments in years 1 and 3 (for a total of eight possible booster sessions), or randomized to not receive additional booster training.

Speed of processing training-This was process-, computer-based training, focused on improving the display speed at which participants could correctly identify increasingly complex displays of visual information. Training involved feedback and practice of perceptual exercises. The exercises focused on speed of processing (stimulus identification), divided attention, selective attention (e.g., divided attention and inhibition), and selective attention with a central discrimination task (Ball, Edwards, \& Ross, 2007; Jobe et al., 2001).

Reasoning training-This training was conducted using paper-and-pencil tasks designed to improve linear problem solving. Training involved practice and feedback on identifying patterns or sequences, such as finding the pattern in a series of repeating letters, or identifying dosage patterns of medications (Jobe et al., 2001; Willis \& Caskie, 2013).

Memory training-This training was conducted using paper-and-pencil tasks designed to teach mnemonic strategies focused on verbal episodic memory. The training involved practice and feedback with organizing materials into meaningful categories that would promote transfer to everyday functioning (e.g., remembering lists of errands, grocery shopping, etc.; Jobe et al., 2001; Rebok et al., 2013).

No-contact control-Participants randomized to this arm came to the study sites for all assessments. No intervention was conducted with this arm.

\section{Measures}

\section{Physical functioning}

Digit symbol copy: Fine motor, visuomotor coordination, and motor speed (Kreiner \& Ryan, 2001) was assessed using Digit Symbol Copy (Wechsler, 1981). After receiving a demonstration and then successfully completing four practice items, participants were asked to copy 93 symbols as quickly as possible into empty boxes below the symbols. Scores were the time it took to complete the task with higher scores reflecting worse performance.

Turn 360 test: Measurement of complex gross motor coordination (i.e., lower limb functioning and dynamic balance) was assessed with the Turn 360 Test (SteinhagenThiessen \& Borchelt, 1999). Participants were asked to turn in a complete circle as quickly and safely as possible while observers recorded the number of steps required to complete each turn. Scores were calculated by taking the average number of steps across two trials, with fewer steps indicating better performance.

Grip strength: Grip strength, a measure of gross motor muscle functioning, was measured using the Jamar hydraulic hand dynamometer by Lafayette Instruments. The handheld dynamometer has been shown to be a reliable and valid instrument in older adults, even in 
those with moderate to severe cognitive impairments (Al Snih et al., 2002; Syddall, Cooper, Martin, Briggs, \& Aihie Sayer, 2003). Two trials of grip strength, recorded in kilograms (kg) and rounded to the nearest whole number, were obtained for the participant's reported dominant hand using a standardized script and protocol (Jobe et al., 2001). The grip strength test was waived if participants reported having hand or arm surgery during the last 3 months, tendonitis, recent worsening of pain, or arthritis. If performed, the two trials were averaged to yield a composite measure of grip strength, with higher scores reflecting greater strength.

\section{Training}

Three separate effects-coded variables were created which compared individuals randomized to the control group $(-1)$ to each individual training group (1). The number of training sessions participants received ranging from 0 (those in the control group) to 18 (those who received all booster sessions) was used as a time-varying measure of dosage (treatment received).

\section{Covariates}

Demographics-Age, years of education $(0-20)$, sex (female $=1$; male $=0$ ), and race (non-White $=1$; or White $=0$ ) were included as covariates as prior work found that older age (Clouston et al., 2013), lower educational attainment (Honjo, Iso, Ikeda, Inoue, \& Tsugane, 2009), female sex (Blankevoort et al., 2013), and non-White race (Rantanen et al., 1998) were predictive of lower physical functioning. Age and education were centered on the grand mean for the sample.

Self-reported physical functioning-The baseline physical functioning subscale of the SF-36 functional health and well-being survey was used to assess perceived physical functioning within the context of daily life (Ware \& Sherbourne, 1992). Perceptions of physical function are indicative of underlying health conditions that may influence both cognitive and physical abilities (Cress et al., 1995). Scores range from 0 to 100, and higher scores indicate better perceived physical functioning. Physical functioning was centered on the sample grand mean.

\section{Analyses}

Chi-square and ANOVAs were conducted to assess if the study arms differed at baseline on the variables of interest (Table 1). To assess change in physical functioning, all three outcome variables were independently standardized at each time point by first subtracting the baseline mean and then dividing by the baseline standard deviation. Time was scaled as a time-varying measure reflecting the number of months since the baseline assessment (i.e., 2month posttest and 1-, 2-, 3-, and 5-year follow-up assessments). Base models were developed for each outcome prior to examining potential training transfer effects using the control arm of the study $(n=698)$. Development of base models involved examining the impact of demographics (age, education, sex, and race) and baseline physical functioning as well as Time $\times$ Covariate interactions, for each outcome. Nonsignificant covariates and interactions were removed from each base model, resulting in one final base model per outcome measure. 
Three separate intention to treat (ITT) mixed-effects analyses assessed the effect of randomization to each individual training arm (speed of processing, reasoning, or memory) vs. the control arm on each outcome without consideration of adherence to the training. Treatment-received mixed-effects analyses were also conducted to account for noncompliance and assess the impact of actual training received (rather than randomization to training arm/ITT analyses) on each outcome using number of training sessions received as a time-varying predictor. The treatment-received models included all participants in the control and respective training group arms. Analytic samples differed by model depending on final outcome and covariates, and are included in Tables 2 through 4 . All models used restricted maximum-likelihood estimation with an unstructured covariance matrix and were conducted with SPSS, version 22. Random-effects estimates are unstandardized and significance was evaluated at $p<0.05$.

\section{Results}

Chi-square and ANOVAs revealed that the study arms did not differ at baseline on the outcomes or covariates.

\section{Digit Symbol Copy Time}

Digit symbol copy base model—Base models developed using the control study arm revealed that being female (est. $=-.321[S E=0.121], p=.008 ; 95 \%$ confidence interval $[\mathrm{CI}]=[-.559,-.083])$ and having better baseline perceived physical functioning (est. $=-$. $011[S E=0.002], p<.001 ; 95 \% \mathrm{CI}=[-0.016,-0.007])$ significantly predicted better Digit Symbol Copy performance across 5 years. There were also significant Age $\times$ Time (est. $<$. $001[S E<.001], p<.001 ; 95 \% \mathrm{CI}=[<0.001,<0.001])$ and Race $\times$ Time (est. $=.003[S E=$ $0.001], p=.01 ; 95 \% \mathrm{CI}=[0.001,0.005])$ interactions, demonstrating that younger and White participants had better performance over time (i.e., less steep declines in performance). Other covariates (education) and interactions (Education $\times$ Time, Sex $\times$ Time, perceived physical function $\times$ Time) were not predictive of changes in Digit Symbol Copy time and were removed from further analyses.

Intention-to-treat (ITT)—No significant training effects were found for any group comparisons (speed of processing training vs. control, reasoning training vs. control, or memory training vs. control) in the ITT analyses.

Treatment-received-Treatment-received models assessed the impact of number of training sessions on Digit Symbol Copy across 5 years after accounting for sex, baseline physical function, Age $\times$ Time and Race $\times$ Time. Results revealed that compared to the control group, more sessions of speed of processing training (est. $=-.005[S E<0.001], p<$. $001 ; 95 \% \mathrm{CI}=[-0.007,-0.002]$ ), reasoning training (est. $=-.009[S E=0.001], p<.001$; $95 \% \mathrm{CI}=[-0.012,-0.006]$ ), and memory training (est. $=-.009[S E=0.001], p<.001 ; 95 \%$ $\mathrm{CI}=-0.012,-0.007]$ ) resulted in better Digit Symbol Copy performance (see Table 2 and Figure 1). 


\section{Turn 360}

Turn 360 base model—Base models developed using the control study arm revealed that non-White race (est. $=-.167[S E=0.064], p=.009 ; 95 \% \mathrm{CI}=-0.292,-0.042]$ ) and higher baseline perceived physical functioning (est. $=-.011[S E=0.001], p<.001 ; 95 \% \mathrm{CI}=$ $[-0.014,-0.009])$ predicted better Turn 360 performance. There was also a significant Age $\times$ Time interaction (est. $=.001[S E<0.001], p<.001 ; 95 \% \mathrm{CI}=[<0.001,0.001])$ which demonstrated that older participants declined at a faster rate. Other covariates (education, sex) and interactions (Education $\times$ Time, Sex $\times$ Time, Race $\times$ Time, Perceived physical functioning $\times$ Time) were not predictive of changes in Turn 360 and were removed from further analyses.

Intention-to-treat (ITT)—No significant training effects were found for any group comparisons (speed of processing training vs. control, reasoning training vs. control, or memory training vs. control) in the ITT analyses.

Treatment-received-Treatment-received models assessed the impact of number of training sessions on Turn 360 across 5 years after accounting for race, baseline physical functioning, and Age $\times$ Time. Results revealed that more sessions of speed of processing training (est. $=-.008[S E=0.003], p=.004 ; 95 \% \mathrm{CI}=[-0.014,-0.003]$ ), reasoning training (est. $=-.010[S E=0.003), p=.001 ; 95 \% \mathrm{CI}=[-0.015,-0.004])$ and memory training (est. $=-.008[S E=0.003), p=.004 ; 95 \% \mathrm{CI}=[-0.013,-0.003])$, predicted better Turn 360 across the 5 years relative to the control group (See Table 3 and Figure 1).

\section{Grip Strength}

Grip strength base model-Base models developed using the control study arm revealed that non-White race (est. $=.378[S E=0.057], p<.001 ; 95 \% \mathrm{CI}=[0.265,0.490]$ ) and higher baseline perceived physical functioning (est. $=.006$ [SE=0.001], $p<.001 ; 95 \% \mathrm{CI}$ $=[0.004,0.008])$ predicted better grip strength. The Age $\times$ Time $($ est. $<.000[S E<0.001], p$ $=.004 ; 95 \% \mathrm{CI}=[<-0.001,<-0.001])$ and Sex $\times$ Time (est. $=-.002[S E=0.001], p=$. $032 ; 95 \% \mathrm{CI}=[-0.005,<0.00])$ interactions revealed that older participants and female participants declined faster over time. Other covariates (education) and interactions (Education $\times$ Time, Race $\times$ Time, Perceived physical function $\times$ Time) were not predictive of changes in grip strength and were removed from further analyses.

Intention-to-treat (ITT)—No significant training effects were found for any group comparisons (speed of processing training vs. control, reasoning training vs. control, or memory training vs. control) in the ITT analyses.

Treatment-received-Treatment-received models assessed the number of training sessions on grip strength across 5 years after accounting for race, baseline physical functioning, and Age $\times$ Time and Sex $\times$ Time interactions. Results revealed that more sessions of reasoning training (est. $=.005[S E=0.002], p=.012 ; 95 \% \mathrm{CI}=[0.001,0.009]$ ) predicted better grip strength across the 5 years relative to controls. Speed of processing training (est. $=.004[S E=0.002], p=.072 ; 95 \% \mathrm{CI}=[<-0.001,0.008]$ ) did not significantly affect grip strength; however, there was a trend, although not significant, for memory 
training (est. $=.004[S E=0.002], p=.046 ; 95 \% \mathrm{CI}=[<0.001,0.008]) ;($ See Table 4 and Figure 1).

\section{Discussion}

To our knowledge, this is the first study to examine the impact of three different cognitive interventions on multiple objective physical functioning measures across 5 years. Current results demonstrate that the number of sessions of speed of processing, reasoning, and memory training were positively related to performance on motor coordination/speed and dynamic balance measures across 5 years in a large sample of community-dwelling older adults. Importantly, the training sessions did not improve physical functioning compared to baseline, but rather, higher doses of cognitive training (i.e., more sessions) mitigated physical functioning declines across five years relative to the control group. These results are in line with Smith-Ray and colleagues' (2013; 2014) work, which found transfer to gait speed with visual distraction and dynamic balance outcomes after 20 to 30 hours of multimodal interventions. Similar to the Timed Up and Go and Walking with Visual Distraction tasks, tests such as Digit Symbol Copy and Turn 360 require complex neuromuscular coordination for successful performance. Because cognitive functioning is closely related to complex motor functions, including motor speed and coordination, gait, and balance (Hausdorff, Yogev, Springer, Simon, \& Giladi, 2005), it is not surprising that cognitive interventions targeting these basic abilities would transfer to Digit Symbol Copy and Turn 360. However, this study provides a key link to the growing research area by addressing limitations identified in prior work such as increased sample sizes and longitudinal data ( $\mathrm{Li}$ et al., 2010; Smith-Ray et al., 2013; Smith-Ray et al., 2014). Given the importance of physical functioning, these far transfer effects are noteworthy.

Additional far transfer effects to everyday functioning have been observed. For example, speed of processing training has demonstrated transfer to performance-based Instrumental Activities of Daily Living (IADL) at immediate posttest (Edwards et al., 2005), self-reported IADLs across 10 years (Rebok et al., 2014), driving safety and mobility across 5 to 6 years (Ball, Edwards, Ross, \& McGwin, 2010; Ross et al., 2016), and maintained health and decreased health expenditures across 5 years (Wolinsky et al., 2010; Wolinsky et al., 2009). Reasoning training transferred to maintained driving safety and mobility across 5 to 6 years (Ball et al., 2010; Ross et al., 2016) as well as self-reported IADLs across 10 years (Rebok et al., 2014). Interestingly, while speed of processing and reasoning training maintained targeted cognitive effects across 10 years, memory training did not demonstrate such maintenance effects on memory although individuals who received memory training did report better IADLs across 10 years (Rebok et al., 2014). Cognitive training programs clearly demonstrate differential maintenance and transfer effects highlighting the importance of carefully examining the specific outcomes, follow-up time periods, and training methods when examining the efficacy of training programs.

All three cognitive interventions (speed of processing, reasoning, and memory training) transferred to maintained Digit Symbol Copy and Turn 360 performance across 5 years; however, only reasoning training demonstrated benefits to grip strength. To our knowledge, this is the first study to examine cognitive training and grip strength, thus prior research for 
comparison is lacking. The physical and cognitive loads of this task are relatively limited as compared to the dynamic and complex motor tasks required by Digit Symbol Copy and Turn 360. Higher order cognitive abilities (i.e., spatial and verbal) have differentially predicted grip strength across 20 years (Sternäng et al., 2015). This may help explain why only reasoning training, which targets higher-order executive function abilities (Jobe et al., 2001), transferred to grip strength. As a relatively simple motor movement, grip strength may be less affected by central processing degradations than more complex motor movements, and at the point in which grip strength is noticeably diminished, central processing integrity may already be significantly compromised. However, further research is needed before these relationships can be fully explained.

Increased interdependence of cognitive, sensory and motor systems appears to occur in later life. Indeed, evidence suggests age-related increases in attentional demands for certain types of movement, including those involving somatosensory information and visual feedback, result in an increased reliance on the prefrontal cortex and basal ganglia for motor control (Seidler et al., 2010). These areas of the brain also appear to be particularly important for higher order cognitive processes and dual-task demands (Hertzog, Kramer, Wilson, \& Lindenberger, 2008; Seidler et al., 2010), but are paradoxically susceptible to earlier and more pronounced age-related declines (Hertzog et al., 2008). Thus, interventions targeting these areas may effectively mitigate age-related functional declines, particularly among functions with cognitive and motor control components. The differential results by outcome measure in the present study lend support to this hypothesis. Digit Symbol Copy and Turn 360 assessments require integration of cognitive and motor abilities, constituting a complex dual-task environment. Moreover, both of these tasks require greater involvement of somatosensory information and visual feedback than does the grip strength test.

The current study, together with prior similar work (Li et al., 2010; Smith-Ray et al., 2013; Smith-Ray et al., 2014; Verghese et al., 2010), clearly demonstrates that cognitive training has the potential to impact physical functioning. Greater amounts of cognitive training across all three interventions resulted in blunted declines in Digit Symbol Copy and Turn 360; however, only reasoning training reduced grip strength declines. This indicates that gross motor functioning may be more difficult to target via cognitive intervention and require higher-order cognitive training programs, possibly due to the low cognitive burden required to perform gross motor tasks.

The exact mechanisms of training transfer are unknown. Proposed mechanisms of these transfer effects range from specific age-related brain changes to a more global deterioration of brain integrity (Clouston et al., 2013). Functional brain imaging has shown stronger and more pervasive brain activation in older adults relative to younger adults when performing simple and complex motor movements (Heuninckx, Wenderoth, Devaere, Peeters, \& Swinnen, 2005). Furthermore, recruitment of cognitive resources increase when performing complex coordination tasks (Buckner, 2004; Cabeza, 2001; Cabeza, Anderson, Locantore, \& McIntosh, 2002; Reuter-Lorenz, 2002) such as complex dual cognitive and physical function tasks (e.g., Turn 360). It is possible that cognitive training may directly or indirectly delay age-related decreases in nervous system processing, which would result in better physical functioning. Furthermore, the improvements in physical function were found even after 
controlling for baseline-reported physical function, reducing the likelihood that such effects are a result of baseline perceptions of physical functioning or well-being. Similarly, prior work has found that speed of processing training effects were not due to socialization, changes in self-efficacy, or other placebo effects using mediational analyses and active control groups (Edwards, Ruva, O'Brien, Haley, \& Lister, 2013; O'Brien et al., 2013; Ross et al., 2016; Sharpe, Holup, Hansen, \& Edwards, 2014).

Given the small magnitude of the effects, in comparison to those found with exercise and rehabilitation programs, we are not suggesting that cognitive training be proposed as the best intervention to improve physical functioning. Rather, these results provide further experimental support for the interconnectedness of cognition and physical functioning across time. In addition, given that the patterns of transfer varied by intervention type, these results provide further evidence that transfer mechanisms may differ by cognitive training program approach. Future work should focus on the mechanisms underlying such transfer effects. Hypothesized mechanisms of such neural plasticity include increased neurogenesis, synaptogenesis, and myelin remodeling (O'Rourke, Gasperini, \& Young, 2014).

Unfortunately, data allowing direct examination of possible mechanisms were not available in the current study and remain unknown. However, taken together, current and prior results provide a compelling argument for future work examining causal processes.

In terms of significant covariates, race, Age $\times$ Time (i.e., over time, older adults had steeper physical function declines), and poorer self-reported baseline physical function were consistent predictors of poor physical function. Non-White participants had worse performance on Digit Symbol Copy but better performance on Turn 360 and grip strength. This is consistent with previous work highlighting the importance of age-related changes in physical function and extends previous work by demonstrating the importance of race in physical function (Clouston et al., 2013). Previous work suggests the impact of race on physical function measures is not consistent across measures (Rantanen et al., 1998), and these findings also demonstrate the complex relationship between race and physical function. The current findings may also elucidate prior work which found that a multicomponent cognitive intervention significantly improved balance with large effect sizes in a sample of Black participants who were at risk of mobility difficulties. In comparison, the current sample was predominantly White and had small effect sizes. It may be that the benefits of cognitive training are enhanced in at-risk or non-White samples, and future work should continue exploring differential intervention effects among various subgroups of older adults (Smith-Ray et al., 2014).

\section{Conclusion}

In conclusion, this study found significant far-transfer effects between three distinct cognitive training interventions and three objective measures of physical functioning over a 5 -year period among healthy older adults. The current study contributes salient information to the literature as the first study to demonstrate that cognitive training may longitudinally affect physical functioning. In addition, this study highlights the importance of considering dosage (treatment-received) effects as well as differential transfer effects to individual domains of physical functioning. 
Future research should continue to explore the interplay between cognitive and physical functioning, particularly interventions that target these areas. Investigators should focus on elucidating the mechanisms behind far-transfer effects, other measures of physical functioning (e.g., gait speed), differential effects of cognitive training programs; and more diverse samples (e.g., older adults with physical functioning impairments). Future work should also examine whether the changes in cognitive performance explain the longitudinal changes in physical functioning. This line of research has important public health implications, given the importance of both cognitive and physical functioning for older adults' independence, health, and well-being.

\section{Acknowledgments}

This work was supported by cooperative agreements (U01AG14260; U01AG14263; U10AG14276; U01AG14282; U01AG14289; U01NR04507; U01NR04508) from the National Institute on Aging and the National Institute of Nursing Research, National Institutes of Health.

\section{References}

Albert SM, Bear-Lehman J, Anderson SJ. Declines in mobility and changes in performance in the instrumental activities of daily living among mildly disabled community-dwelling older adults. The Journals of Gerontology Series A: Biological Sciences and Medical Sciences. 2015; 70(1):71-77. DOI: $10.1093 /$ gerona/glu088

Alfaro-Acha A, Snih SA, Kuo Y-F, Markides KS, Ottenbacher KJ. Handgrip strength and cognitive decline in older Mexican Americans. The Journals of Gerontology Series A: Biological Sciences and Medical Sciences. 2006; 61:859-865. DOI: 10.1093/gerona/61.8.859

Al Snih S, Markides KS, Ray L, Ostir GV, Goodwin JS. Handgrip strength and mortality in older Mexican Americans. Journal of the American Geriatrics Society. 2002; 50:1250-1256. DOI: 10.1046/j.1532-5415.2002.50312.x [PubMed: 12133020]

Anstey KJ, Lord SR, Williams P. Strength in the lower limbs, visual contrast sensitivity, and simple reaction time predict cognition in older women. Psychology and Aging. 1997; 12(1):137-144. DOI: 10.1037/0882/7974.12.1.137 [PubMed: 9100274]

Atkinson HH, Rapp S, Williamson JD, Lovato J, Absher JR, Gass M, Espeland MA. The relationship between cognitive function and physical performance in older women: Results from the women's health initiative memory study. The Journals of Gerontology Series A: Biological Sciences and Medical Sciences. 2010; 65:300-306. DOI: 10.1093/gerona/glp149

Ball KK, Edwards JD, Ross LA. The impact of speed of processing training on cognitive and everyday functions. The Journals of Gerontology Series B: Psychological Sciences and Social Sciences. 2007; 62(Special Issue 1):19-31.

Ball KK, Edwards JD, Ross LA, McGwin G Jr. Cognitive training decreases motor vehicle collision involvement of older drivers. Journal of the American Geriatrics Society. 2010; 58(11):2107-2113. DOI: 10.1111/j.1532-5415.2010.03138.x [PubMed: 21054291]

Blankevoort CG, Scherder EJA, Wieling MB, Hortobágyi T, Brouwer WH, Geuze RH, van Heuvelen JG. Physical predictors of cognitive performance in healthy older adults: A cross-sectional analysis. Plos One. 2013; 8:1-9. DOI: 10.1371/journal.pone.0070799

Bohannon RW. Hand-grip dynamometry predicts future outcomes in aging adults. Journal of Geriatric Physical Therapy. 2008; 31:3-10. DOI: 10.1519/00139143-200831010-00002 [PubMed: 18489802]

Bridenbaugh SA, Kressig RW. Motor cognitive dual tasking: Early detection of gait impairment, fall risk and cognitive decline. Zeitschrift fur Gerontologie und Geriatrie. 2015; 48(1):15-21. DOI: 10.1007/s00391-014-0845-0 [PubMed: 25633391]

Buchman AS, Boyle PA, Leurgans SE, Barnes LL, Bennett DA. Cognitive function is associated with the development of mobility impairments in community-dwelling elders. American Journal of Geriatric Psychiatry. 2011; 19:571-580. DOI: 10.1097/JGP.0b013e3181ef7a2e [PubMed: 21606900] 
Buckner RJ. Memory and executive function in aging and AD: Multiple factors that cause decline and reserve factors that compensate. Neuron. 2004; 44(1):195-208. DOI: 10.1016/j.neuron. 2004.09.006 [PubMed: 15450170]

Cabeza R. Cognitive neuroscience of aging: Contributions of functional neuroimaging. Scandinavian Journal of Psychology. 2001; 42:277-286. DOI: 10.1111/1467-9450.00237 [PubMed: 11501741]

Cabeza R, Anderson ND, Locantore JK, McIntosh AR. Aging gracefully: Compensatory brain activity in high-performing older adults. Neuroimage. 2002; 17:1394-1402. DOI: 10.1006/nimg. 2002.1280 [PubMed: 12414279]

Clouston SAP, Brewster P, Kuh D, Richards M, Cooper R, Hardy R, Hofer SM. The dynamic relationship between physical function and cognition in longitudinal age cohorts. Epidemiological Review. 2013; 35:33-50. DOI: 10.1093/epirev/mxs004

Collins LM, Trail JB, Kugler KC, Baker TB, Piper ME, Mermelstein RJ. Evaluating individual intervention components: Making decisions based on the results of a factorial screening experiment. Translational Behavioral Medicine. 2014; 4(3):238-251. DOI: 10.1007/ s13142-013-0239-7 [PubMed: 25264464]

Cress ME, Schechtman KB, Mulfrow CD, Fiatarone MA, Gerety MB, Buchner DM. Relationship between physical performance and self-perceived physical function. Journal of the American Geriatrics Society. 1995; 43(2):93-101. DOI: 10.1111/j.1532-5415.1995.tb06372.x [PubMed: 7836655]

Desjardins-Crépeau L, Berryman N, Vu TT, Villalpando JM, Kergoat MJ, Li KZ, Bherer L. Physical functioning is associated with processing speed and executive functions in community-dwelling older adults. The Journals of Gerontology Series B: Psychological Sciences and Social Sciences. 2014; 69:837-844. DOI: 10.1093/geronb/gbu036

Doumas M, Rapp MA, Krampe RT. Working memory and postural control: Adult age differences in potential for improvement, task priority, and dual tasking. Journal of Gerontology:

PSYCHOLOGICAL SCIENCES. 2009; 64B(2):193-201. DOI: 10.1093/geronb/gbp009

Edwards JD, Ruva CL, O'Brien JL, Haley CB, Lister JJ. An examination of mediators of the transfer of cognitive speed of processing training to everyday functional performance. Pscyhology and aging. 2013; 28(2):314-321. DOI: 10.1037/a0030474

Edwards JD, Wadley VG, Vance DE, Wood K, Roenker DL, Ball KK. The impact of speed of processing training on cognitive and everyday performance. Aging \& Mental Health. 2005; 9(3): 262-271. DOI: 10.1080/13607860412331336788 [PubMed: 16019280]

Gale CR, Allerhand M, Aihie Sayer A, Cooper C, Deary IL. The dynamic relationship between cognitive function and walking speed: The English Longitudinal Study of Ageing. Age. 2014; 2014:1-11. DOI: 10.1007/s11357-014-9682-8

García-Peña C, García-Fabela LC, Gutiérrez-Robledo LM, García-González JJ, Arango-Lopera VE, Pérez-Zepeda MU. Handgrip strength predicts functional decline at discharge in hospitalized male elderly: A hospital cohort study. PloS one. 2013; 8:1-7. DOI: 10.1371/journal.pone.0069849

Gleeson M, Sherrington C, Keay L. Exercise and physical training improve physical function in older adults with visual impairments but their effects on falls is unclear: A systematic review. Journal of Physiotherapy. 2014; 60:130-135. DOI: 10.1016/j.phys.2014.06.010 [PubMed: 25066935]

Hausdorff JM, Yogev G, Springer S, Simon ES, Giladi N. Walking is more like catching than tapping: Gait in the elderly as a complex cognitive task. Experimental Brain Research. 2005; 164(4):541548. DOI: 10.1007/s00221-005-2280-3 [PubMed: 15864565]

Hertzog C, Kramer AF, Wilson RS, Lindenberger U. Enrichment effects on adult cognitive development. Psychological Science in the Public Interest. 2008; 9(1):1-65. DOI: 10.1111/j. 1539-6053.2009.01034.x [PubMed: 26162004]

Heuninckx S, Wenderoth N, Devaere F, Peeters R, Swinnen SP. Neural basis of aging: The penetration of cognition into action control. The Journal of Neuroscience. 2005; 25(29):6787-6796. DOI: 10.1523/JNEUROSCI.1263-05.2005 [PubMed: 16033888]

Honjo K, Iso H, Ikeda A, Inoue M, Tsugane S. Education level and physical functional limitations among Japanese community residents- gender difference in prognosis from stroke. BMC Public Health. 2009; 9(131):1-8. DOI: 10.1186/1471-2458-9-131 [PubMed: 19121216] 
Jobe JB, Smith DM, Ball K, Tennstedt SL, Marsiske M, Willis SL, Kleinman K. ACTIVE: A cognitive intervention trial to promote independence in older adults. Controlled Clinical Trials. 2001; 22(4): 453-479. DOI: 10.1016/S0197-2456(01)00139-8 [PubMed: 11514044]

Kluger A, Gianutsos JG, Golomb J, Ferris SH, George AE, Franssen E, Reisberg B. Patterns of motor impairment in normal aging, mild cognitive decline, and early Alzheimer's Disease. Journals of Gerontology: Series B, Psychological Sciences and Social Sciences. 1997; 52(1):P28-P39. DOI: 10.1093/geronb/52B.1.P28

Kreiner DS, Ryan JJ. Memory and motor skill components of the WAISIII Digit Symbol-Coding subtest. The Clinical Neuropsychologist. 2001; 15(1):109-113. DOI: 10.1076/clin.15.1.109.1906 [PubMed: 11778571]

Li KZ, Roudaia E, Lussier M, Bherer L, Leroux A, McKinley PA. Benefits of cognitive dual-task training on balance performance in healthy older adults. Journals of Gerontology: Series A, Biological Sciences and Medical Sciences. 2010; 65(12):1344-1352. DOI: 10.1093/gerona/glq151

Lord SR, Castell S, Corcoran J, Dayhew J, Batters B, Shan A, Williams B. The effect of group exercise on physical functioning and falls in frail older people living in retirement villages: A randomized, controlled trial. Journal of the American Geriatrics Society. 2003; 51:1685-1692. DOI: 10.1046/j. 1532-5415.2003.51551.x [PubMed: 14687345]

Nieto ML, Albert SM, Morrow LA, Saxton J. Cognitive status and physical function in older AfricanAmericans. Journal of the American Geriatrics Society. 2008; 56:2014-2019. DOI: 10.1111/j. 1532-5415.2008.01938.x [PubMed: 18811612]

O'Brien JL, Edwards JD, Maxfield ND, Peronto CL, Williams VA, Lister JJ. Cognitive training and selective attention in the aging brain: An electrophysiological study. Clinical Neurophysiology. 2013; 124(11):2198-2208. DOI: 10.1016/j.clinph.2013.05.012 [PubMed: 23770088]

O'Rourke M, Gasperini R, Young KM. Adult myelination: Wrapping up neuronal plasticity. Neural Regeneration Research. 2014; 9(13):1261-1264. DOI: 10.4103/1673-5374.137571 [PubMed: 25221576]

Painter P, Stewart AL, Carey S. Physical functioning: Definitions, measurement, and expectations. Advances in Renal Replacement Therapy. 1999; 6(2):110-123. DOI: 10.1016/ S1073-4449(99)70028-2 [PubMed: 10230878]

Rantanen T, Guralnik JM, Leveille S, Izmirlian G, Hirsch R, Simonsick E, Fried LP. Racial differences in muscle strength in disabled older women. Journal of Gerontology: Biological Sciences. 1998; 53A(5):B355-B361. DOI: 10.1093/gerona/53A.5.B355

Rebok GW, Ball KK, Guey LT, Jones RN, Kim H-Y, King JW, Willis SL. Ten year effects of the Advanced Cognitive Training for Independent and Vital Elderly cognitive training trial on cognition and everyday functioning in older adults. Journal of the American Geriatrics Society. 2014; 62:16-24. DOI: 10.1111/jgs.12607 [PubMed: 24417410]

Rebok GW, Langbaum J, Jones RN, Gross AL, Parisi JM, Spira AP, Brandt J. Memory training in the ACTIVE study: How much is needed and who benefits? Journal of Aging and Health. 2013; 25(8S):21s-42s. DOI: 10.1177/0898264312461937 [PubMed: 23103452]

Reuter-Lorenz PA. New visions of the aging mind and brain. Trends in Cognitive Sciences. 2002; 6(9): 394-400. DOI: 10.1016/s1364-6613(02)01957-5 [PubMed: 12200182]

Rosano C, Simonsick EM, Harris TB, Kritchevsky SB, Brach J, Visser M, Newman AB. Association between physical and cognitive function in healthy elderly: The health, aging, and body composition study. Neuroepidemiology. 2005; 24:8-14. DOI: 10.1159/00081043 [PubMed: 15459503]

Ross LA, Edwards JD, O'Connor ML, Ball KK, Wadley VG, Vance DE. The transfer of cognitive speed of processing training to older adults' driving mobility across 5 years. Journals of Gerontology Series B: Psychological Sciences and Social Sciences. 2016; 71(1):87-97. DOI: 10.1093/geronb/gbv022

Seidler RD, Bernard JA, Burutolu TB, Fling BW, Gordon MT, Gwin JT, Lipps DB. Motor control and aging: Links to age-related brain structural, functional, and biochemical effects. Neuroscience and Biobehavioral Reviews. 2010; 34(5):721-733. DOI: 10.1016/j.neubiorev.2009.10.005 [PubMed: 19850077] 
Sharpe C, Holup AA, Hansen KE, Edwards JD. Does self-efficacy affect responsiveness to cognitive speed of processing training? Journal of Aging and Health. 2014; 26(5):789-806. DOI: $10.1177 / 0898264314531615$

Smith-Ray RL, Hughes SL, Prohaska TR, Little DM, Jurivich DA, Hedeker D. Impact of cognitive training on balance and gait in older adults. The Journals of Gerontology Series B: Psychological Sciences and Social Sciences. 2013; 70:357-366. DOI: 10.1093/geronb/gbt097

Smith-Ray RL, Makowski-Woidan B, Hughes SL. A randomized trial to measure the impact of a community-based cognitive training intervention on balance and gait in cognitively intact black older adults. Health Education \& Behavior. 2014; 41(Supplement 1):62S-69S. DOI: 10.1177/1090198114537068 [PubMed: 25274713]

Steinhagen-Thiessen, E., Borchelt, M. Morbidity, medication, and functional limitations in very old age. In: Baltes, PB., Mayer, KU., editors. The Berlin Aging Study: Aging from 70 to 100. Cambridge University Press; Cambridge, UK: 1999.

Sternäng O, Reynolds CA, Finkel D, Ernsth-Bravell M, Pedersen NL, Dahl Aslan AK. Grip strength and cognitive abilities: Associations in old age. Journals of Gerontology. Series B: Psychological Sciences and Social Sciences. 2015; epub ahead of print. doi: 10.1093/geronb/gbv017

Syddall H, Cooper C, Martin F, Briggs R, Aihie Sayer A. Is grip strength a useful single marker of frailty? Age and Ageing. 2003; 32:650-656. DOI: 10.1093/ageing/afg111 [PubMed: 14600007]

van Iersel MB, Kessels RPC, Bloem BR, Verbeek ALM, Olde Rikkert MGM. Executive functions are associated with gait and balance in community-living elderly people. The Journals of Gerontology Series A: Biological Sciences and Medical Sciences. 2008; 63:1344-1349. DOI: 10.1093/gerona/ 63.12.1344

Verghese J, Mahoney J, Ambrose AF, Wang C, Holtzer R. Effect of cognitive remediation on gait in sedentary seniors. The Journal of Gerontology Series A: Biological Sciences and Medical Sciences. 2010; 65(12):1338-1343. DOI: 10.1093/gerona/glq127

Ware JE Jr. Sherbourne CD. The MOS 36-item short-form health survey (SF-36). I. Conceptual framework and item selection. Medical Care. 1992; 30(6):473-483. [PubMed: 1593914]

Wechsler, D. Manual for the Wechsler Adult Intelligence Scale-Revised. Psychological Corporation; New York: 1981.

Willis SL, Caskie GIL. Reasoning training in the ACTIVE study: How much is needed and who benefits? Journal of Aging and Health. 2013; 25(8S):43S-64S. DOI: 10.1177/0898264313503987 [PubMed: 24385639]

Willis SL, Tennstedt S, Marsiske M, Ball KK, Elias J, Koepke KM, Wright E. Long-term effects of cognitive training on everyday functional outcomes in older adults. Journal of the American Medical Association. 2006; 296(23):2805-2814. DOI: 10.1001/jama.296.23.2805 [PubMed: 17179457]

Wolinsky FD, Mahncke H, Vander Weg MW, R. M, Unverzagt FW, Ball KK, Tennstedt S. Speed of processing training protects self-rated health in older adults: Enduring effects observed in the mulit-site ACTIVE randomized controlled trial. International Psychogeriatrics. 2010; 22(3):470478. DOI: 10.1017/S1041610209991281 [PubMed: 20003628]

Wolinsky FD, Mahncke HW, Kosinski M, Unverzagt FW, Smith DM, Jones RN, Tennstedt SL. The ACTIVE cognitive training trial and predicted medical expenditures. BMC Health Services Research. 2009; 9(1):109.doi: 10.1186/1472-6963-9-109 [PubMed: 19558724] 
a.
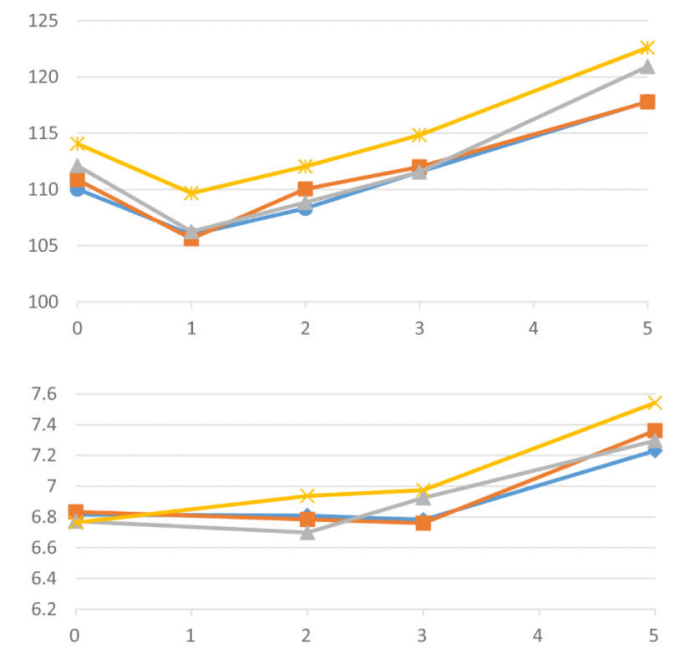

c.

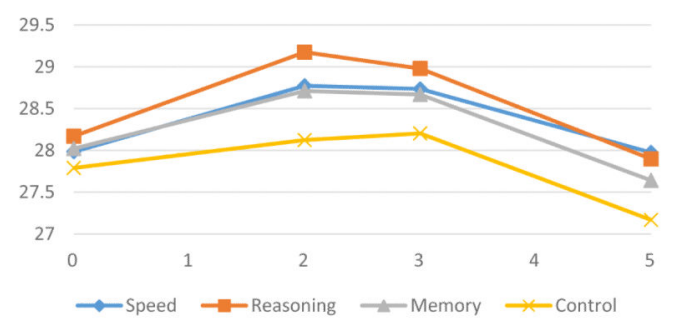

Figure 1.

The impact of three cognitive interventions on physical functioning across five years.

Note: (a) Digit Symbol Copy after accounting for age, sex, race, and self-reported physical function. (b) Turn 360 after accounting for age, race, and self-reported physical function. (c) Grip strength after accounting for age, sex, race, and self-reported physical function. Lower scores indicate better performance for Digit Symbol Copy and Turn 360, and higher scores indicate better performance for grip strength. 


\section{Table 1}

Demographics by Study Arm

\begin{tabular}{lcccc}
\hline Variable & $\begin{array}{c}\text { Speed of } \\
\text { processing } \\
\text { training arm } \\
(\boldsymbol{n}=\mathbf{7 0 2})\end{array}$ & $\begin{array}{c}\text { Reasoning } \\
\text { training arm } \\
(\boldsymbol{n}=\mathbf{6 9 4})\end{array}$ & $\begin{array}{c}\text { Memory } \\
\text { training arm } \\
(\boldsymbol{n}=703)\end{array}$ & $\begin{array}{c}\text { No-contact } \\
\text { control arm } \\
(\boldsymbol{n}=\mathbf{6 9 8})\end{array}$ \\
\hline $\begin{array}{l}\text { Digit Symbol Copy, } M \\
(S D)\end{array}$ & $102.5(30.3)$ & $104.8(34.2)$ & $104.6(34.9)$ & $108.1(73.2)$ \\
Age, $M(S D)$ & $73.4(5.8)$ & $73.6(5.8)$ & $73.5(6.0)$ & $74.1(6.1)$ \\
Education, $M(S D)$ & $13.7(2.7)$ & $13.5(2.7)$ & $13.6(2.7)$ & $13.4(2.7)$ \\
Female Sex, n $(\%)$ & $538.0(76.6)$ & $532.0(76.7)$ & $537.0(76.4)$ & $514.0(73.6)$ \\
Non-White, n $(\%)$ & $179.0(25.5)$ & $193.0(27.8)$ & $179.0(25.5)$ & $195.0(27.9)$ \\
$\begin{array}{l}\text { SF-36 Physical } \\
\text { functioning, } M(S D)\end{array}$ & $69.7(24.1)$ & $67.4(24.2)$ & $69.1(23.5)$ & $68.9(24.6)$ \\
$\begin{array}{l}\text { Digit Symbol Copy, } M \\
(S D)\end{array}$ & $102.5(30.3)$ & $104.8(34.2)$ & $104.6(34.9)$ & $108.1(73.2)$ \\
$\begin{array}{l}\text { Turn 360, } M(S D) \\
\text { Grip strength, } M(S D)\end{array}$ & $24.0(8.3)$ & $24.4(8.4)$ & $24.0(8.4)$ & $24.0(8.1)$ \\
\hline
\end{tabular}

Note. Chi-square and ANOVAs revealed that study arms did not differ on any variables at baseline. 


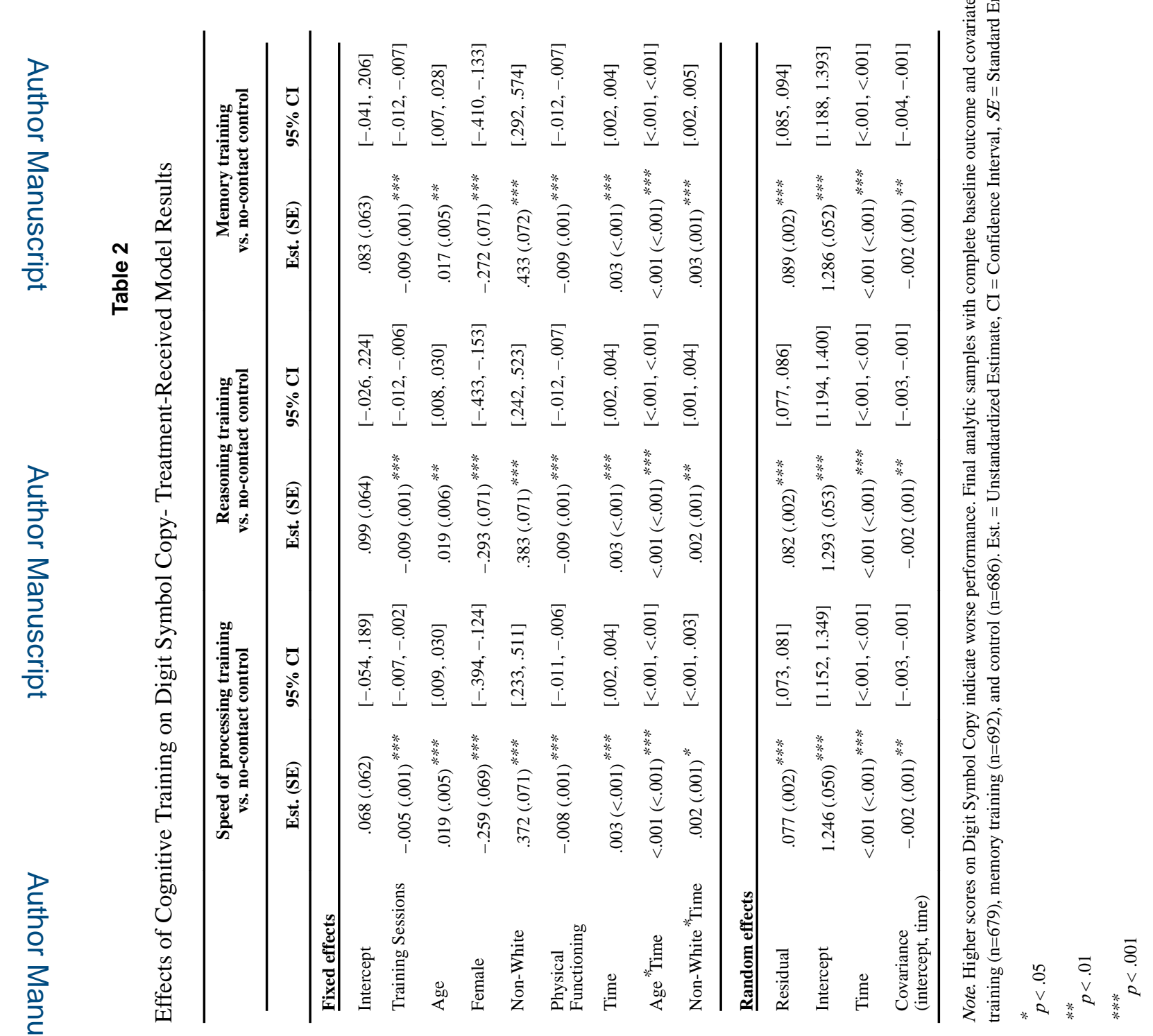




\section{롤 \\ ฏ.}

בิ

焉

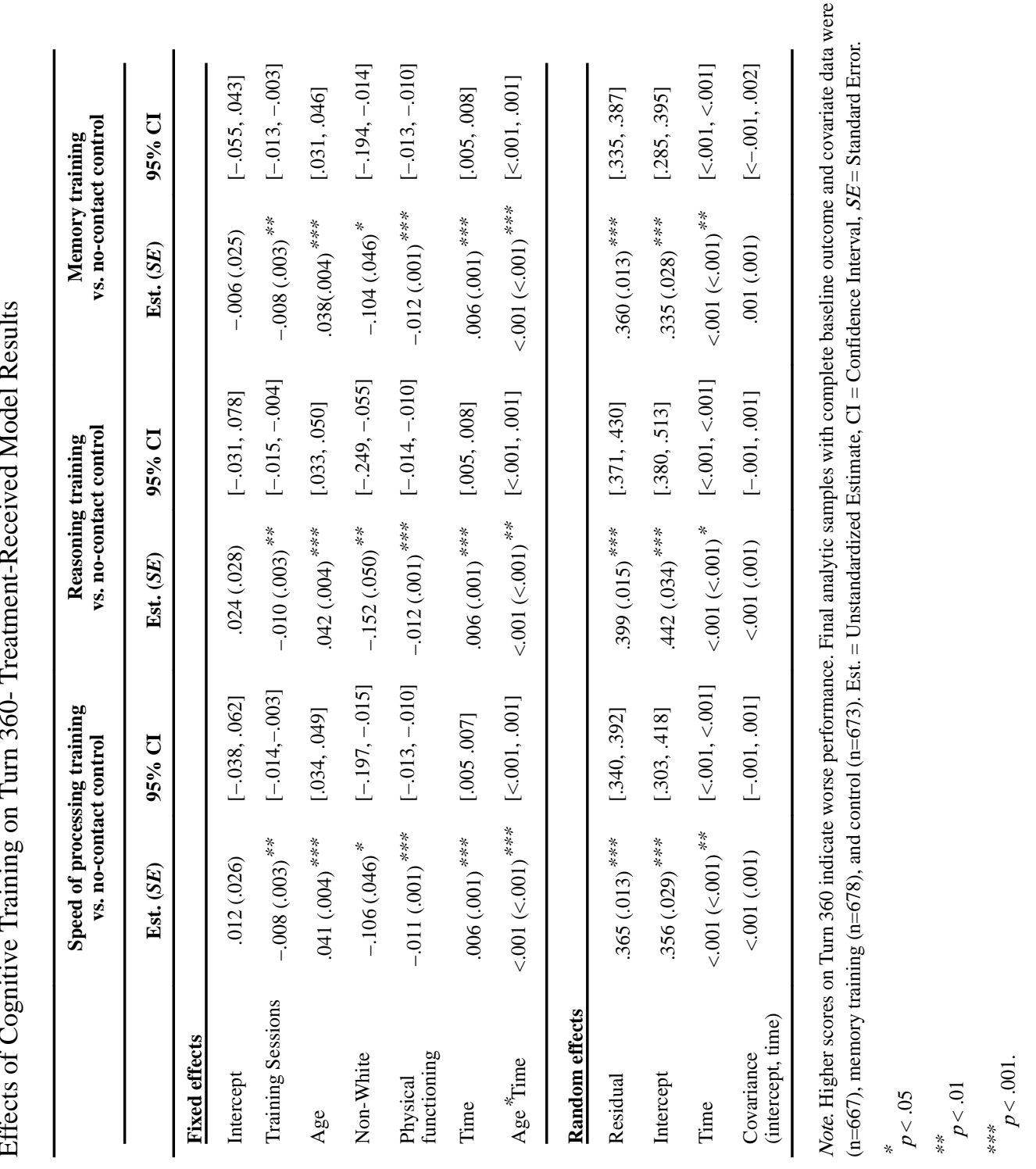

J Aging Health. Author manuscript; available in PMC 2019 March 01. 


\section{롷 \\ 골}

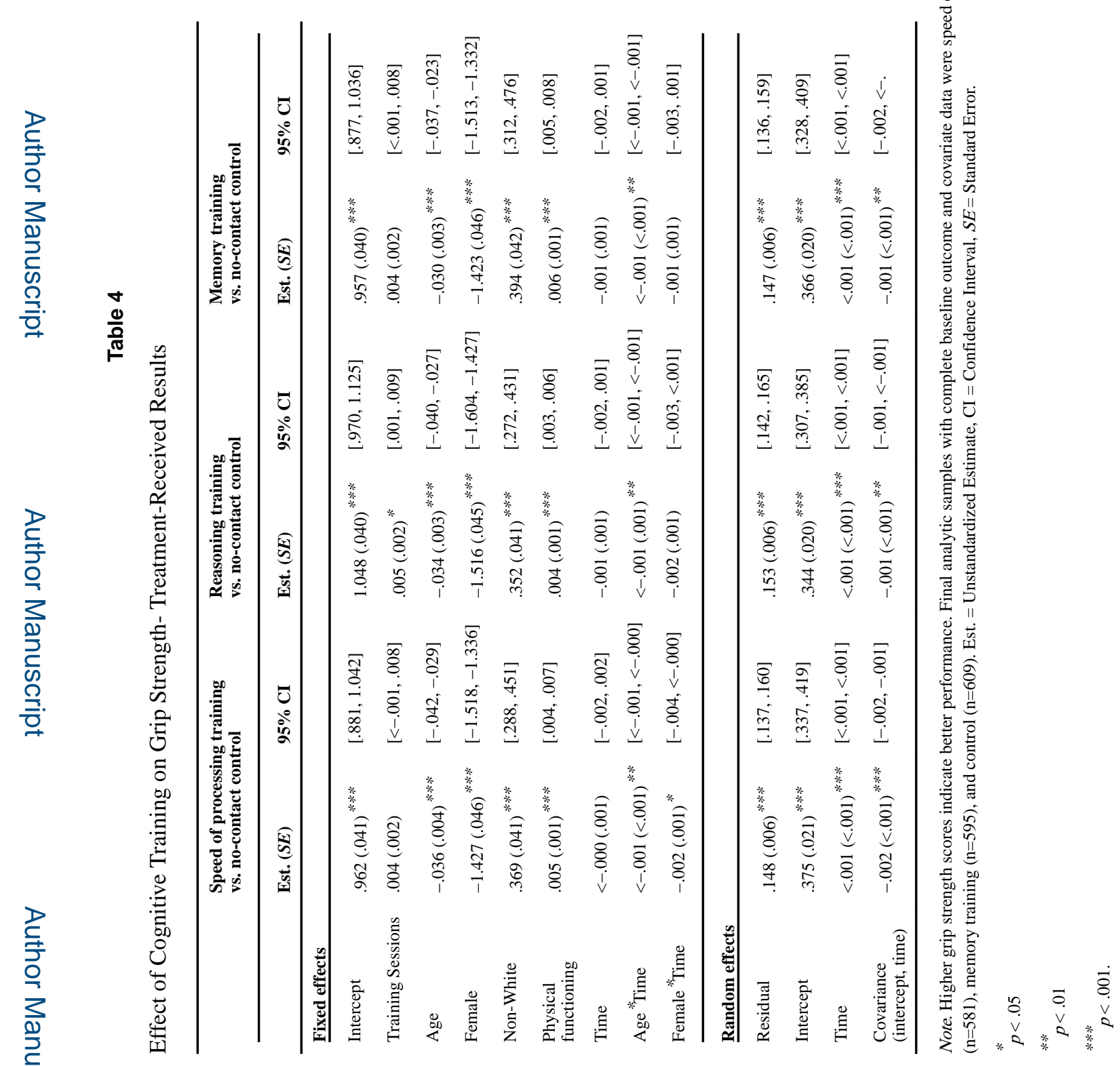

J Aging Health. Author manuscript; available in PMC 2019 March 01. 